\title{
Estimating the trend of the French BSE epidemic over six birth cohorts through the analysis of abattoir screening in 2001 and 2002
}

\author{
Claude LA BonnARDiÈrE ${ }^{\mathrm{a}, \mathrm{b}}$, Didier CAlAVAS ${ }^{\mathrm{c}}$, David ABRIAL ${ }^{\mathrm{a}}$, \\ Eric MORIGNAT ${ }^{\mathrm{c}}$, Christian DUCROT $^{\mathrm{a} *}$ \\ a Unité d'Épidémiologie Animale, INRA Theix, 63122 Saint-Genès-Champanelle, France \\ b Unité de Virologie et Immunologie Moléculaires, INRA, Domaine de Vilvert, \\ 78352 Jouy-en-Josas Cedex, France \\ c Unité d'Épidémiologie, AFSSA Lyon, 31 av. T. Garnier, 69364 Lyon Cedex 07, France
}

(Received 8 September 2003; accepted 29 January 2004)

\begin{abstract}
A bovine spongiform encephalopathy (BSE) testing programme at the abattoir started in 2001 in France. A total of 5281293 bovines were tested in 2001 and 2002; 87 were found positive in 2001 - 37 per million (95\% CI 30-46) -, whereas only 71 in $2002-24$ per million (95\% CI 1930). Logistic regression models were run to compare the prevalence of BSE on successive birth cohorts, using a pair-wise method of controlling for age at testing; the prevalence on the first one, determined on animals slaughtered in 2001, was compared to the prevalence on the following one determined on animals slaughtered in 2002. Five models were performed in order to compare the birth cohorts preceding and following the months of June 1993 (i.e. July 92-June 93 birth cohort compared to July 93-June 94 birth cohort) (8.5 years old cattle), June 1994 (7.5 years old cattle), June 1995 (6.5 years old cattle), June 1996 (5.5 years old cattle) and June 1997 (4.5 years old cattle). The models were adjusted for the production type of cattle and the test used. The results showed a significant increase (OR $=2.31,95 \%$ CI 1.08-4.9) of the BSE prevalence between the July 93-June 94 and July 94-June 95 cohorts, and then a significant decrease over the next two birth cohorts; the July 95-June 96 birth cohort was significantly less affected than the July 94-June 95 one (OR = $0.46,95 \%$ CI $0.27-0.78$ ), and the July 96-June 97 birth cohort was significantly less affected than the July $95-$ June 96 one $(\mathrm{OR}=0.17,95 \%$ CI $0.07-0.37)$. The increase in BSE prevalence between the July 93-June 94 and July 94-June 95 cohorts was in agreement with modelling studies, but needs to be confronted to the data on fallen stock at the national level. The decrease in BSE prevalence on the birth cohorts born after June 1995 was in agreement with the findings on the fallen stock in the western part of France and matches the implementation of the removal of specified risk materials (SRM) and dead animals from the processing of meat and bone meal (MBM) since June 1996.
\end{abstract}

\section{BSE / prevalence / epidemiology / abattoir screening / logistic regression}

\section{INTRODUCTION}

The first case of BSE was evidenced in 1991 [17] in France, and 108 others were discovered until June 2000 with the clinical surveillance [10]. Since then different complementary surveillance programmes have been organised [1], based on rapid tests [22]; they showed that the clinical surveillance alone misses a great proportion of diseased animals [15, 20]. This is the reason why a comprehensive system is now implemented to detect BSE cases, including clinical surveillance, test on dead cattle (fallen

\footnotetext{
* Corresponding author: ducrot@clermont.inra.fr
} 
stock), and systematic screening at the abattoir; the tests are carried out on cattle aged two years and more (over 30 months old before July 2001 at the abattoir). Retrospective clinical surveys have shown that two thirds of the animals testing positive among the fallen stock had shown probable or definite clinical signs of BSE before death, whereas only $7 \%$ of those testing positive at the abattoir have had signs evocating those of BSE before death [7]. This suggests that a great part of the cases discovered at the fallen stock were clinically diseased whereas most of those at the abattoir were at the pre clinical stage of the disease.

A major effort has been made to control BSE, which is transmissible to humans $[5,18]$. However, the ban of meat and bone meal (MBM) in cattle foodstuff has proven insufficient to control the overall contamination process of the disease in Great Britain as well as in other countries like France where 752 cases have been detected on animals born after the ban on the feeding of MBM to cattle (July 1990) (called NAIF for Nés Après l'Interdiction des Farines animales), up to July 1st, 2003 [1]. In France, MBM was still authorised until the end of 2000 for monogastric species, and it has been hypothesised that NAIF cases could be due to the cross contamination of cattle feed with foodstuff dedicated to monogastric species and containing MBM. So, complementary measures were taken in France in June 1996 in order to secure the MBM intended for monogastric food; carcasses from dead animals as well as specified risk materials (SRM) have been removed from MBM processing since then [10]. Given the length of the incubation period, we are now able to evaluate the efficiency of this control measure by estimating the current trend of the epidemic.

A comprehensive analysis of the data concerning fallen stock was performed [21] over a three year period in western France where a pilot study started in June 2000 [20]. It showed that, on animals dead or sub- jected to euthanasia, the prevalence of positive BSE tests decreased for animals born after June 1995. Our paper presents the analysis of the trend of the epidemic on the whole metropolitan French territory through the analysis of the abattoir screening over a two year period. We used the same global methodology as for the fallen stock screening [21] so that the results may be compared.

\section{MATERIALS AND METHODS}

\subsection{Reference population}

The reference population was composed of all cattle sent to the abattoir for human consumption between January 1,2001 and December 31, 2002, aged two years (30 months between January and June 2001) or more at the time of slaughter. These animals were subjected to a screening test defined by the European Union (EU) regulation No. 999/2001/CE [16]. In France, the age limit was lowered to 24 months old whereas the regulation threshold is 30 months. Emergency slaughtered cattle were not concerned because a French regulation of December 2000 requires that they be sent to the fallen-stock whatever the reason of the emergency, accident or disease [10]. Bovines (5281 293) were included in the abattoir screening during the period of interest, respectively 2352284 in 2001 and 2929009 in 2002. Individual identification of French cattle has been required since the nineteen-seventies, and individual data are collected at birth, so only $1.9 \%$ of the slaughtered cattle in our dataset had missing values on the date of birth, with $45 \%$ of them being foreign cattle slaughtered in French slaughter houses.

\subsection{Definition of BSE cases}

BSE cases were test positive animals detected through the screening programme at the abattoir, with either of the two tests: Prionics-check (Roche Diagnostic, Mannheim, Germany) or Platelia BSE (Biorad, 
Table I. Description of the five models carried out to compare successive birth cohorts tested in 2001 and 2002 .

\begin{tabular}{|c|c|c|c|c|c|c|c|}
\hline \multirow[b]{2}{*}{ Model } & \multicolumn{3}{|c|}{$\begin{array}{c}\text { Cohort A } \\
\text { Year of test: } 2001\end{array}$} & \multicolumn{3}{|c|}{$\begin{array}{c}\text { Cohort B } \\
\text { Year of test: } 2002\end{array}$} & \multirow{2}{*}{$\begin{array}{c}\text { Age (years) } \\
\text { Range (midpoint) }\end{array}$} \\
\hline & Birth Cohort & $\mathrm{Nb}$ tests & $\begin{array}{c}\text { Positives } \\
\mathrm{Nb}(\%)\end{array}$ & Birth Cohort & $\mathrm{Nb}$ tests & $\begin{array}{c}\text { Positives } \\
\mathrm{Nb}(\%)\end{array}$ & \\
\hline 1 & July 92-June 93 & 123494 & $0(<0.001)$ & July 93-June 94 & 151174 & 7 (0.005) & $7.5-9.5(8.5)$ \\
\hline 2 & July 93-June 94 & 159415 & $9(0.006)$ & July 94-June 95 & 194851 & $27(0.014)$ & $6.5-8.5(7.5)$ \\
\hline 3 & July 94-June 95 & 197280 & $36(0.018)$ & July 95-June 96 & 243451 & $22(0.009)$ & $5.5-7.5(6.5)$ \\
\hline 4 & July 95-June 96 & 242030 & $35(0.014)$ & July 96-June 97 & 291046 & $7(0.002)$ & $4.5-6.5(5.5)$ \\
\hline 5 & July 96-June 97 & 297886 & $5(0.002)$ & July 97-June 98 & 355120 & $2(0.0006)$ & $3.5-5.5(4.5)$ \\
\hline
\end{tabular}

Marnes la Coquette, France), and confirmed positive at the National Reference Laboratory (AFSSA Lyon, France) with western blot [19] or immunohistochemistry [9]. A total of $158 \mathrm{BSE}$ cases were included in the study, respectively 87 in 2001 and 71 in 2002.

\subsection{Statistical analysis}

First, the overall trend of the BSE prevalence at the abattoir was analysed by comparing the percentage of positive animals in 2001 and 2002, with the hypothesis that the age distribution, the geographical origin and the production type of the slaughtered animals did not vary significantly between years. Confidence intervals for prevalence estimates were calculated assuming a Poisson distribution for the BSE frequencies (reviewed in [8]). Also, we analysed the distribution of the percentage of positive animals per year of birth, in order to identify the birth cohorts the most at risk in 2001 and 2002.

Then, in order to evaluate the trend in the exposure of cattle to the BSE agent, the prevalence of BSE at the time of slaughter was analysed for different birth cohorts, defined by the seasonal year of birth from July 1 to June 30 . The prevalence of BSE in a given birth cohort, slaughtered in 2001, was compared to the prevalence of BSE in the following birth cohort slaughtered in 2002, so that the comparison of the prevalence of BSE in the different birth cohorts was done on animals of the same age.

More precisely, five models were carried out (Tab. I), in order to compare the birth cohorts preceding and following the months of June 1993 (i.e. the July 92-June 93 birth cohort compared to the July 93-June 94 birth cohort), June 1994, June 1995, June 1996 and June 1997. Models 1 and 5 were known to have a smaller statistical power than the others, since the number of positive animals was very small on animals younger than five years old and older than eight years old. Nevertheless, they were run in order to discuss the trend observed on the other models. Furthermore, not a single animal was found positive in 2001 in the July 92-June 93 birth cohort; in order to be able to run the model, we created a fictitious dairy positive cow in this category.

The study unit was the slaughtered animal and the outcome the result of the rapid test after confirmation of test positives by the National Reference Laboratory (positive versus negative). The data were analysed with multivariate non-conditional logistic regressions [4]. The main explanatory variable was the birth cohort and its contribution was measured with the odds 
Table II. The results of the models comparing successive birth cohorts (one tested in 2001 and the following one in 2002).

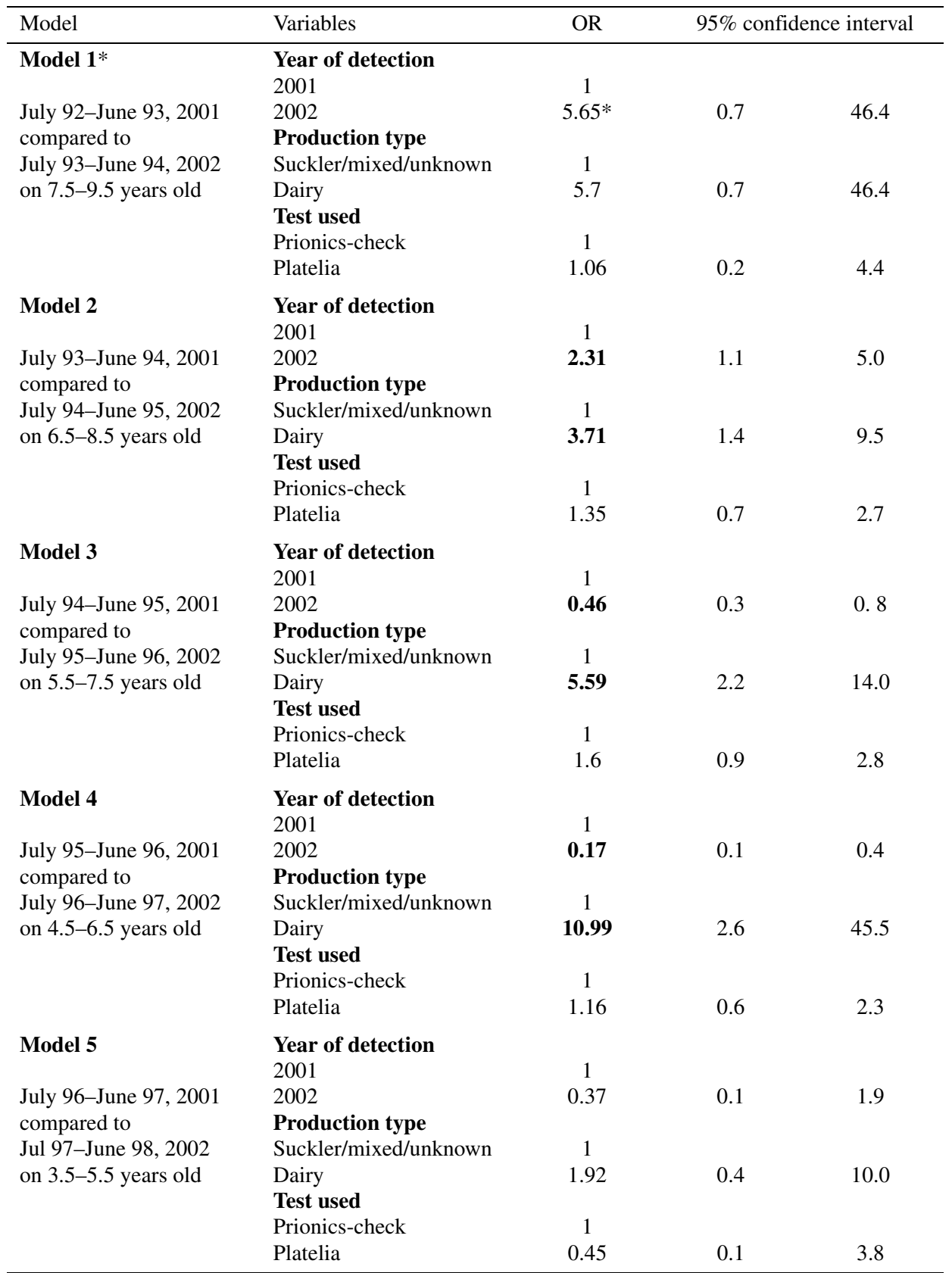

* Adjusted OR in a model where a fictitious positive dairy cow was attributed to 2001 to be able to run the model.

Odds ratio significantly different from 1 at $P<0.05$ are printed in bold type. 
Table II. Continued.

\begin{tabular}{lccccc}
\hline Model & 1 & 2 & 3 & 4 & 5 \\
\hline Null deviance & 183.10 & 733.98 & 1152.54 & 877.69 & 174.21 \\
(degrees of freedom) & 274667 & 354265 & 440730 & 533075 & 653005 \\
Residual deviance & 175.24 & 716.96 & 1120.93 & 828.52 & 170.99 \\
(degrees of freedom) & 274664 & 354262 & 440727 & 533072 & 653002 \\
\hline
\end{tabular}

ratio. The analysis was adjusted for the production type of the animal (defined according to the breed type and classified in dairy versus beef suckler, mixed or unknown), and the type of test used (Prionics-check versus Platelia). The region of the farm was not included in the model because the number and the type of cattle originating from each region did not vary over the years. Data handling was done using ACCESS (Microsoft Access 97, (C) 1989-1997 Microsoft Corporation) and the statistical analysis using SAS software (SAS 8.1, Logistic procedure, (C) 1999-2000 SAS Institute Inc.).

\section{RESULTS}

The overall BSE prevalence at the time of slaughter in France decreased from 37 positives per million tests $(95 \%$ CI 30 46 ) in 2001 to 24 positives per million tests (95\% CI 19-30) in 2002. The analysis of the percentage of positives per year of birth (not shown) indicated that the years of birth the most at risk for being positive were the same in 2001 and 2002, that is to say 1995 and secondly 1994.

The results of the five logistic regressions carried out by comparing two by two the successive cohorts at the same age at slaughter, are presented in Table II. Three of them showed significant odds ratios for the birth cohort effect. The comparison of the birth cohorts showed a significant increase $(\mathrm{OR}=2.31,95 \%$ CI 1.1-4.9) in the BSE prevalence between the July 93-June 94 (detected in 2001) and July 94-June 95 (2002) cohorts, on animals of about 7.5 years old (Model 2). Then a significant decrease of the BSE prevalence occurred over the next two birth cohorts: compared to the July 94 June 95 birth cohort, the following one (July 95-June 96) was significantly less affected $(\mathrm{OR}=0.46,95 \%$ CI $0.3-0.8)$, the animals tested being around 6.5 years old (Model 3). Also, the July 96-June 97 birth cohort was significantly less affected than the July 95-June 96 birth cohort (OR = $0.17,95 \%$ CI $0.1-0.4$ ), the animals tested being around 5.5 years old (Model 4). Models 1 and 5 did not show any significant effect, neither for the birth cohort effect, nor for the production type and test variables.

The production type, dairy versus beef cattle, was linked significantly to the BSE prevalence for models 2 to 4 ; even if the estimate of the odds ratio varied between models, from 3.7 to 10.9 , their confidence intervals were in the same range of values. Because the type of test (Platelia /Prionicscheck) was linked significantly to the result of the test on the 2002 data, this factor was forced into the models, but it did not show a significant effect.

\section{DISCUSSION}

\subsection{Population of concern}

It has been shown that the percentage of BSE cases found either through the Mandatory Reporting System or through the different active surveillance programmes (on fallen stock and at the abattoir) varies with time and space [12, 20]. It might be possible that some animals with mild clinical signs were sent to the abattoir, instead of being declared to the mandatory reporting system, since it has been found that about $7 \%$ of the animals tested positive at the abattoir had 
shown some clinical signs suggestive of BSE [7]. This is the reason why the results from the abattoir testing programme have to be compared to the results found on fallen stock, to verify if the same trend was observed.

The testing programme at the abattoir has been implemented for Human health reasons and there is no doubt about exhaustiveness. However, during the first semester of 2001, apart from the abattoir slaughter for human consumption and in the aim of market support following the BSE crisis in the autumn of 2000, 179086 animals were slaughtered and destroyed without being tested. These animals, which represented $16 \%$ of the overall population slaughtered during that period, had a worse body condition score on average than the animals sent for consumption [14], resulting in a possible underestimation of the real prevalence of BSE on tested cattle. In order to take into account this possible bias, we reran the logistic models on the subpopulation of animals sent to the abattoir during the second semester of 2001 and 2002 only, when no market support was in force. The results (not shown) evidenced the same trend, but were not significant because of a lack of power of this partial analysis. This tends to indicate that the possible escape route-intentionally or not-during the first semester of 2001 did not represent a bias to analyse the trend in the epidemic. The fact that fewer cattle were tested in 2001 compared to 2002 was explained partly by the temporary destruction programme in 2001 and also by the drop of the age threshold for the screening test after June 2001.

\subsection{Diagnosis}

Two diagnostic tests have been used, depending on the local diagnostic laboratories; this might be a source of bias in the results. A formal comparison of the performance of these tests (sensitivity and specificity) was made in 1999 in the frame of a European evaluation in laboratory condi- tions showing no evidence of performance difference [22], but this assessment was made only on clinically diseased and disease free animals. However, all the French cases detected with one or the other of the two tests have always been confirmed by the other one so far, showing no difference in their ability to detect cases. Also, all positive results have been confirmed at the National Reference Laboratory. However, in order to take into account possible differences between the tests, we forced the variable in the models.

\subsection{Statistical method}

The comparison of the prevalence of BSE positive animals of a given age during successive years was based on the hypotheses that the distribution of the age at contamination and the incubation period were assumed to be constant over two years of time in the studied area. If they were true, then the differences in BSE prevalence between cohorts might be attributed to differences in the level of infection over years. There is no reason to think that the age at contamination changed during such a short period of time. Concerning the incubation, the 1996 control measures - removal of SRM and cadavers from the meat and bone meal - might have produced a dilution effect in the contaminated substance. If the length of the incubation depends on the infectious titre, the probability for an animal exposed to the BSE agent to declare the disease at a given age might have varied over time; this dose effect function is not known for cattle. If this had occurred, with a contamination level maintained constant and an increase in the length of the incubation, we would have observed an increase in the age at clinical onset and thus a rise of the percentage of positives over the following years. The fact that the global trend is a decrease in BSE prevalence for the different cohorts following those of 1995 tends to indicate that this phenomenon, if it happened, 
was outdone by the decrease in contamination.

The age structure and the geographical origin of the population sent to the abattoir were very stable from one year to another. These variables can hence hardly be confounders for the analysis of the trend of the epidemic. This is the reason why we did not include the geographical origin of the animal in the models, even if it is known to be a risk factor [11, 20, 23]. It helped to keep the study as powerful as possible. However, we made an adjustment for the production type of cattle which is highly related to the BSE risk [15, 26], first because the culling rates differ between the two types, second because we cannot exclude that the BSE incubation period varies between dairy and beef cows. Another modelling approach might have been to build a single model with the entire slaughtered population and incorporating interaction variables between the year of test of birth cohorts to account for the postulate that the effect of the year of test depended on the birth cohorts being tested; comparing the odds of being a cow of a given birth cohort slaughtered in 2001 to those of a cow of the following birth cohort slaughtered in 2002 would have given the estimates of the risk increase/ reduction between cohorts. We carried out this model that gave the same estimates for the odds ratios. However, in order to be able to compare our results with the study carried out on fallen stock with the same exact method, and to simplify the explanation of the method, we preferred to present the five sub-models.

\subsection{Results}

The results showed a decrease in the BSE prevalence at the abattoir in 2002 compared to 2001. Animals between 24 and 30 months old were not tested during the first semester of 2001; based on the 2002 data, this might represent approximately 138000 animals that should have been all negatives. The correction for this bias still gives a much higher proportion of positive animals in 2001 (35 positive per million tests), compared to 2002 (24 per million). Moreover, given that the prevalence rate at the abattoir during the first semester of 2001 has been underestimated due to the economical measures of the removal of nearly 200000 animals, the real decrease might have been even higher.

The decrease was consistent with the global trend of the crude BSE prevalence in France: since the beginning of the systematic screening of cattle aged 24 months and over at death or slaughter, the crude monthly prevalence proportion of BSE (number of BSE cases over the number of animals included in the different programmes) decreased regularly, from about $0.13 \%$ in the summer of 2001 to $0.04 \%$ o in the spring of 2003 [6].

The birth cohorts the most at risk for being positive at the abattoir were the same for cattle tested in 2001 and 2002. A stable level or a slight decrease in the BSE contamination over the years would have produced a shift in the mode of the year of birth the most at risk between 2001 and 2002, given that the slaughtered population has roughly the same age distribution over the years. The absence of a shift suggested that the July 94-June 95 birth cohort might represent a peak in the observed level of BSE infection in France. This was confirmed with the logistic models. The analysis of the prevalence in the different birth cohorts was performed in such a way that the compared birth cohorts had the same range of age at the abattoir. The statistically significant results showed an increase in the BSE prevalence between the July 93-June 94 and July 94-June 95 birth cohorts, and then a decrease over the two following cohorts. Although not significant, the results of models 1 and 5 had the same trend as those of the other models, with a tendency to a high increase between the July 92-June 93 cohort and the following one (Model 1), and a decrease between the July 95-June 96 cohort and the following one (Model 5). The result of model 5 was even underesti- 
mated since we created a false positive animal to be able to run it. The observed peak in the infection on the July 94-June 95 cohort was in agreement with the results of a mathematical model [13] carried out on the French data. This peak of infection might be the second one in France, since a first one might have occurred previously, in the late nineteen-eighties, according to the results of mathematical models [13, 24]. The first peak has not been evidenced since the surveillance programme was not efficient enough at that time; furthermore, we were not able to study it with our dataset since it was too far in the past; nearly all animals were dead or culled already.

Our results found on animals sent for slaughtering in France were in agreement with the trend of the epidemic found in western France on dead animals, animals subjected to euthanasia or sent for emergency slaughter [21]. The same methodology was used in this previous study; however, the analysis was limited to the western part of France that represents one third of the total French cattle population, and was carried out on three successive testing periods between 2000 and 2002. The decrease started at the same period (between the July 94-June 95 and July 95-June 96 birth cohorts), with the same intensity. More precisely, when comparing the July 94-June 95 and July 95-June 96 cohorts, our model gave an odds ratio of 0.46 for the risk of BSE whereas the two odds ratios obtained on that point from the study on fallen stock - three years of testing were compared were 0.37 and 0.27 . For the comparison of the July 95-June 96 and July 96-June 97 cohorts, we observed an odds ratio of 0.17 in our study and it was 0.21 in the study on fallen stock. A difference between the two studies was observed on the period preceding July 1994; between the July 93-June 94 and July 94-June 95 cohorts, we observed a significant increase in the prevalence of positive animals (an odds ratio of 2.3) whereas the study of the fallen stock in western France showed the same prevalence between the two cohorts. The signif- icant increase in our study was reinforced by the same tendency (not significant) observed during the previous period. One possible explanation is that the decrease in the infection process started earlier in western France. In fact people were more aware of the disease in this region than in any other part of France, since the French BSE epidemic started in this region and has been higher there than elsewhere; it might be possible that more stringent control measures of BSE transmission were taken earlier than required by law in this region. Our results need to be compared to the data on fallen stock on the whole French bovine population when available, in order to check if the possible transfer of some BSE cases from one detection system to another (clinical surveillance and fallen stock screening versus abattoir screening) might create a bias in the results. This is probably not the case given the similarity of our results for each cohort comparison with those of fallen stock in western France.

Our results showed that the exposure to the BSE agent decreased for animals born during and after June 1995, which was one year before the implementation of the ban of animal cadavers and SRM in MBM in France. The results of mathematical models carried out on the age at infection both in Great Britain and France [3, 24] as well as the age at clinical onset in the pathological trials carried out in Great Britain [25] tend to show that most infected animals might be contaminated at a young age; more precisely, the best fit in the models correspond to an infection between six and eighteen months of age. On this basis, the decrease of the infection starting on the July 95-June 96 cohort matches with the setting out of new control measures in June 1996. These results were also in agreement with the increasing average age of the BSE cases detected since 1999 in the different programmes pooled altogether, reflecting a decrease in the exposure roughly five years before $[1,2]$. However, the results of mathematical models carried out on the period preceding the 
implementation of these complementary control measures have already estimated a decrease of the exposure of cattle to BSE in France starting in 1994 [13, 24], i.e. independent from the effectiveness of the measure introduced in 1996. The decrease of the BSE prevalence on cattle born after June 1995 is probably due to the conjunction of an underlying decrease of the exposure and the beneficial effect of the removal of MRS and animal cadavers from MBM intended for monogastric foodstuff since June 1996. So we cannot precisely measure the effectiveness of this measure.

It is hard to draw any simple conclusion from our results concerning the risk for humans, but it is important to assess it. The risk for the consumer depends indeed on different parameters, among others the control measures in force for food safety in France, such as the removal of the SRM from the food chain and the screening tests at the abattoir, that interact with the size of the epidemic, and also the part of the exposure due to imported food, that might have been the major factor in the past; these parameters have varied over time during the last ten years. The effect on the human risk of the secondary peak of BSE that we observed in the July 94-June 95 cohort, following a probable huge peak on cohorts born at the end of the nineteen-eighties and remained mostly undetected, has to be analysed in the light of these different parameters.

\section{ACKNOWLEDGEMENTS}

The authors would like to thank the Direction Générale de l'Alimentation that provided the data of the surveillance programme at the slaughterhouse, as well as the veterinary practitioners, the local veterinary laboratories and the county veterinary services in charge of the logistics of the surveillance programmes.

\section{REFERENCES}

[1] AFSSA, L'ESB en France - Synthèse sur l'évolution de l'épizootie à partir des données disponibles au $1^{\text {er }}$ janvier 2003, 16 p. (2003)
http://www.afssa.fr/ftp/afssa/actu/RapportESB 040203.pdf [consulted 22 August 2003].

[2] AFSSA Lyon, Épidémiosurveillance de l'ESB en France - Tableau de bord au 01/07/03, 22 p. (2003) http://www.afssa.fr/ftp/basedoc/ TBjuin2003.pdf [consulted 22 August 2003].

[3] Anderson R.M., Donnelly C.A., Ferguson N.M., Woolhouse M.E., Watt C.J., Udy H.J., MaWhinney S., Dunstan S.P., Southwood T.R., Wilesmith J.W., Ryan J.B., Hoinville L.J., Hillerton J.E., Austin A.R., Wells G.A., Transmission dynamics and epidemiology of BSE in British cattle, Nature 382 (1996) 779 788.

[4] Breslow N.E., Day N.E., Statistical methods in cancer research: The design and analysis of cohort studies, International agency for research on cancer, World health organization, Lyon, 1987, 406 p.

[5] Bruce M.E., Will R.G., Ironside J.W., McConnell I., Drummond D., Suttle A., McCardle L., Chree A., Hope J., Birkett C., Cousens S., Fraser H., Bostock C.J., Transmission to mice indicate that "new variant" CJD is caused by the BSE agent, Nature 389 (1997) 498-501.

[6] Calavas D., Morignat E., Cazeau G., Ducrot C., ESB: une synthèse de l'épidémiologie en France, Bulletin des Groupements Techniques Vétérinaires (2003) 23-30.

[7] Cazeau G., Ducrot C., Collin E., Desjouis G., Calavas D., Analysis of BSE cases detected by active surveillance to determine their clinical status before death and the reasons for their non-notification by clinical surveillance, Vet. Rec. 154 (2004) 133-136.

[8] Ceresta, Aide-mémoire pratique des techniques statistiques, Revue de Statistique Appliquée - Special issue 34, Lavoisier Ed., Paris, 1986.

[9] Debeer S.O.S., Baron T., Bencsik A.A., Immunohistochemistry of PrPsc within bovine spongiform encephalopathy brain samples with graded autolysis, J. Histochem. Cytochem. 49 (2001) 1519-1524.

[10] DGAL, Site d'information sur l'encephalopathie spongiforme bovine, http://www. agriculture.gouv.fr/esbinfo/esbinfo.htm [consulted 22 August 2003].

[11] Doherr M.G., Heim D., Vandevelde M., Spatial clustering of Swiss bovine spongiform encephalopathy (BSE) cases, in: The 9th Symposium of the International Society for Veterinary Epidemiology and Economics, 2000, p. 3. 
[12] Doherr M.G., Heim D., Fatzer R., Cohen C.H., Vandevelde M., Zurbriggen A., Targeted screening of high-risk cattle populations for BSE to augment mandatory reporting of clinical suspects, Prev. Vet. Med. 51 (2001) 3-16.

[13] Donnelly C.A., Likely size of the French BSE epidemic, Nature 408 (2000) 787-788.

[14] Ducrot C., Maillot M., Morignat E., Gasqui P., Buquet B., Calavas D., Que nous ont appris les tests réalisés en abattoir depuis janvier 2001 sur la situation épidémiologique de l'ESB en

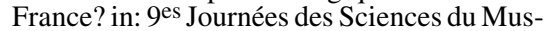
cle et Technologies de la Viande, 2002, pp. 193-194.

[15] Ducrot C., Roy P., Morignat E., Baron T., Calavas D., How the surveillance system may bias the results of analytical epidemiological studies on BSE: prevalence among dairy versus beef suckler cattle breeds in France, Vet. Res. 34 (2003) 185-192.

[16] European Commission, Regulation (EC) No. 999/2001 of the European Parliament and of the Council of 22 May 2001 laying down rules for the prevention, control and eradication of certain transmissible spongiform encephalopathies (2001) http://europa.eu.int/eur-lex/ pri/en/oj/dat/2001/1_147/1_14720010531 en00010040.pdf [consulted $2 \overline{2}$ August 2003].

[17] Gouello L., Analyse épidémiologique du premier cas d'encéphalopathie spongiforme bovine en France, Epidémiologie et Santé Animale 19 (1991) 63-70.

[18] Hill A.F., Desbruslais M., Joiner S., Sidle K.C., Gowland I., Collinge J., Doey L.J., Lantos P., The same prion strain causes vCJD and BSE, Nature 389 (1997) 448-450.

[19] Madec J.-Y., Belli P., Calavas D., Baron T., Efficiency of Western blotting for the specific immunodetection of proteinase K-resistant prion protein in BSE diagnosis in France, Vet. Rec. 146 (2000) 74-76.

[20] Morignat E., Ducrot C., Roy P., Baron T., Vinard J.-L., Biacabe A.G., Madec J.-Y., Bencsik A., Debeer S., Eliazsewicz M., Calavas D., Targeted surveillance to assess the prevalence of BSE in high-risk populations in western France and the associated risk factors, Vet. Rec. 151 (2002) 73-77.

[21] Morignat E., Ducrot C., Roy P., Cohen C.H., Calavas D., Analysis of the prevalence of BSE at time of death to estimate the current trend of the epizootic in western France, Vet. Rec. (in press).

[22] Moynagh J., Schimmel H., Tests for BSE evaluated. Bovine spongiform encephalopathy, Nature 400 (1999) 105.

[23] Stevenson M.A., Wilesmith J.W., Ryan J.B., Morris R.S., Lawson A.B., Pfeiffer D.U., Lin D., Descriptive spatial analysis of the epidemic of bovine spongiform encephalopathy in Great Britain to June 1997, Vet. Rec. 147 (2000) 379-384.

[24] Supervie V., Costagliola D., The unrecognized french BSE epidemic, Vet. Res. 35 (2004) 349-362.

[25] Wells G.A., Hawkins S.A., Green R.B., Austin A.R., Dexter I., Spencer Y.I., Chaplin M.J., Stack M.J., Dawson M., Preliminary observations on the pathogenesis of experimental bovine spongiform encephalopathy (BSE): an update, Vet. Rec. 142 (1998) 103106.

[26] Wilesmith J.W., Ryan J.B., Stevenson M.A., Morris R.S., Pfeiffer D.U., Lin D., Jackson R., Sanson R.L., Temporal aspects of the epidemic of bovine spongiform encephalopathy in Great Britain: holding-associated risk factors for the disease, Vet. Rec. 147 (2000) 319 325. 